\title{
LANDFRIEDEN ALS KONZEPT UND REALITÄT KOLLEKTIVER SICHERHEIT IM HEILIGEN RÖMISCHEN REICH
}

\section{Landfriedenswahrung und Verfassungsentwicklung}

Wenn im Folgenden die Landfriedenswahrung im Heiligen Römischen Reich als Konzept kollektiver Sicherheit diskutiert wird, dann konzentriert sich in dieser Thematik geradezu exemplarisch das Spezifische an der deutschen Verfassungsentwicklung in Spätmittelalter und Früher Neuzeit. Dass gerade in diesem Kontext einige der zentralen Begriffe wie Landfrieden, Einung, Bund (etwa ob ligue oder confédération) oder auch der Begriff »Verfassung « ${ }^{1}$ selbst entweder gar nicht oder nur schwer ins Französische zu übersetzen sind, verweist allerdings darauf, dass die komplexen Strukturen im Reich nur bedingt Entsprechungen in Frankreich oder auch in anderen europäischen Staaten aufzuweisen haben. Die neuere Forschung hat in jüngster Zeit vielfach belegt, dass dies kein Problem der neuesten Historiographiegeschichte ist, sondern dass schon in der Frühen Neuzeit auf französischer Seite die Schwierigkeiten wuchsen, die Komplexität des Heiligen Römischen Reiches noch kategorial zu erfassen; im Verlauf des 18. Jahrhunderts resultierte aus dieser Diskrepanz schließlich ein weitgehendes Unverständnis für das komplexe föderale Gebilde jenseits des Rheins ${ }^{2}$. Im Folgenden sei die These

1 Sowohl die Konzeption von »Verfassungsgeschichte« als politischer Strukturgeschichte wie auch zentrale Begrifflichkeiten aus dem föderativen Erbe des Alten Reiches sind in der französischen Forschung kaum rezipiert worden. Vgl. die instruktiven Bemerkungen von Olivier BÉAUD, La notion de pacte fédératif. Contribution à une théorie constitutionnelle de la Fédération, in: Jean-François KervéGAN, Heinz Mohnhaupt (Hg.), Gesellschaftliche Freiheit und vertragliche Bindung in Rechtsgeschichte und Philosophie - Liberté sociale et lien contractuel dans l'histoire du droit et la philosophie, Frankfurt a.M. 1999 (Studien zur europäischen Rechtsgeschichte, 120), S. 197-270, hier S. 249.

2 Zur frühneuzeitlichen Wahrnehmung v.a. Martin WREDE, Das Reich und seine Geschichte in den Werken französischer Staatsrechtler und Historiker des 18. Jahrhunderts, in: Francia 27 (2000), S. 177-211; DERS., »L'État de l'Empire empire«? Die französische Historiographie und das Reich im Zeitalter Ludwigs XIV. -Weltbild, Wissenschaft und Propaganda, in: Matthias SchneTtger (Hg.), Imperium Romanum - Irregulare Corpus - Teutscher Reichs-Staat. Das Alte Reich im Verständnis der 
vertreten, dass es gerade die spezifischen Regelungen zur Landfriedenswahrung im Reich gewesen sind, die diese föderalen Verfassungsstrukturen des Reiches erfordert und verfestigt haben; sie entfalteten auch deshalb solche Nachhaltigkeit, weil Prinzipien föderaler Landfriedenswahrung auch auf andere »sicherheitsrelevante « Problemfelder wie soziale Unruhen, territoriale Expansion oder die Konfessionsfrage ausgedehnt wurden. Die im Rahmen der Landfriedenswahrung im Reich strukturell bedingte Notwendigkeit, einen Status quo zu definieren und die Stände kollektiv für die Wahrung dieses Status quo in die Pflicht zu nehmen, wurde nicht nur zum föderalen Gegengewicht gegen territoriale Expansion der Mächtigen im Reich, sondern zum fundamentalen Strukturmerkmal des Reiches. Beides aber bedingte sich wechselseitig: Ein System kollektiver Sicherheit, das den Status quo zementierte, schützte einerseits die Mindermächtigen und erhielt andererseits damit auch die Komplexität des Reiches aller wachsenden Dysfunktionalität zum Trotz aufrecht.

»Landfrieden « bezeichnet seit dem Mittelalter eine auf Gewaltverzicht gegründete, räumlich organisierte und ursprünglich zeitlich befristete Friedensordnung. Frieden soll dabei im Wesentlichen dadurch erreicht und gewahrt werden, dass an Stelle der Selbsthilfe (»Faustrecht«) zur Durchsetzung jeweiliger Rechtsansprüche oder zur Ahndung von Unrecht rechtliche Regelungen und entsprechende Gerichts- oder Schiedsinstanzen verbindlich gemacht werden ${ }^{3}$. Im Unterschied zu den westeuropäischen Monarchien, die in England oder Frankreich ein weitgehendes Fehdeverbot und damit autoritativ den Landfrieden durchzusetzen vermochten, ließ sich im Heiligen Römischen Reich eine wirksame Landfriedensgesetzgebung allein aufgrund königlichen

Zeitgenossen und der Historiographie, Mainz 2002, S. 89-110; umfassend jetzt Guido BRAUN, La connaissance du Saint-Empire en France du baroque aux Lumières (16431756), München 2010; WREDE und BRAUN betonen jeweils die zunehmende kategoriale Entfremdung seit dem späten 17. Jahrhundert. In dieser Kontinuität steht erkennbar auch die jüngere französische Historiographie zumindest zur Frühen Neuzeit, vgl. Christophe Duhamelle, Das Alte Reich im toten Winkel der französischen Historiographie, in: Schnettger, Imperium Romanum, S. 207-219; Cornel Zwierlein, Das Imperium im blinden Fleck des Empire: Die Zerstörung des Alten Reiches durch Napoleon 1806, in: Christine Roll, Matthias SchnetTGER (Hg.), Epochenjahr 1806 ? Das Ende des Alten Reichs in zeitgenössischen Perspektiven und Deutungen, Mainz 2008, S. 61-98, v.a. S. 62, Anm. 4.

${ }^{3}$ Hans-Jürgen BECKER, Landfrieden I, in: Lexikon des Mittelalters, München 1991, Bd. 5, Sp. 1657f.; Ekkehard KAUFMANN, Landfrieden I (Landfriedensgesetzgebung), in: Handwörterbuch zur deutschen Rechtsgeschichte, hg. von Alfred ERLER, Ekkehard Kaufmann, Berlin 1979, Bd. 3, Sp. 1451-1465; Heinz HolzHAuER, Landfrieden II (Landfrieden und Landfriedensbruch), in: ibid., Sp. 1465-1485; Horst CARL, Landfrieden, in: Enzyklopädie der Neuzeit, hg. von Friedrich JÄGER, Stuttgart 2008, Bd. 7, Sp. 493-500. 
Gebots nicht erfolgreich realisieren. Seit dem 13. Jahrhundert scheiterten entsprechende Aktivitäten des Königtums, auch wenn am Anspruch, dass die Wahrung des Landfriedens eine genuin königliche bzw. kaiserliche Aufgabe sei, festgehalten wurde ${ }^{4}$. Gerade aufgrund der Aufrechterhaltung dieses Anspruches aber wurde die faktische Friedlosigkeit im Reich immer stärker als Versagen des Königtums in seiner zentralen Aufgabe wahrgenommen und kritisiert. Als Folge wurde zwangsläufig die Friedenswahrung dezentralisiert und regionalisiert, indem sich weltliche Herrschaftsträger - in der Regel befristet - in Schwureinungen zusammenschlossen, um die interterritoriale Friedenswahrung selbst in die Hand zu nehmen ${ }^{5}$. Landfriedenswahrung wurde deshalb im Reich in Form von Organisationen kollektiver Sicherheit realisiert.

An dieser Stelle also kommen die zahlreichen Einungen (Schwurgenossenschaften) im Reich ins Spiel, die das Spätmittelalter prägten. Sie seien hier nicht in extenso aufgefächert, sondern es sei nur daran erinnert, dass es spätestens seit Otto von Gierke eine deutsche verfassungsgeschichtliche Tradition gibt, diese Einungen zur dominierenden Organisationsform im spätmittelalterlichen Heiligen Reich zu stilisieren - durchaus zeitweilig mit nationalistischen Konnotationen ${ }^{6}$. Dies ist schon deshalb wenig überzeugend, weil die Stadt als Schwureinung der Bürgergemeinde 7 , Adelsgesellschaften oder Ritterorden, wie sie Werner Paravicini und Andreas Ranft untersucht haben $^{8}$, Städtebünde ${ }^{9}$ und schließlich auch beschworene Gemeinden, wie sie

${ }^{4}$ Dies ist das zentrale Thema von Heinz AngermeIER, Königtum und Landfriede im deutschen Spätmittelalter, München 1966.

5 Aus der Fülle der Spezialliteratur sei nur auf die jüngste Monographie verwiesen: Matthias FAHRNER, Der Landfrieden im Elsass. Recht und Realität einer interterritorialen Friedensordnung im späten Mittelalter, Marburg 2007, S. 113-292.

${ }^{6}$ Otto vON GIERKE, Das deutsche Genossenschaftsrecht, 4 Bde., Berlin 1868-1913. Die starke deutschrechtliche Betonung bei Gierke hat die Rezeption systematischer Anknüpfungspunkte jenseits Deutschlands eher behindert: Otto Gerhard OEXLE, Otto von Gierkes »Rechtsgeschichte der deutschen Genossenschaft«. Ein Versuch wissenschaftsgeschichtlicher Rekapitulation, in: Notker HAMMERSTEIN (Hg.), Deutsche Geschichtswissenschaft um 1900, Stuttgart 1988, S. 193-217.

${ }^{7}$ Ernst PITZ, Bürgereinung und Städteeinung. Studien zur Verfassungsgeschichte der Hansestädte und der deutschen Hanse, Köln, Weimar, Wien 2001.

${ }^{8}$ Holger KRUSE, Werner PARAVICINI, Andreas RAnFt (Hg.), Ritterorden und Adelsgesellschaften im spätmittelalterlichen Deutschland, Frankfurt a.M. u.a. 1991; Andreas RANFT, Adelsgesellschaften. Gruppenbildung und Genossenschaft im spätmittelalterlichen Reich, Sigmaringen 1994.

${ }_{9}^{9}$ Eva-Marie DistLeR, Städtebünde im deutschen Spätmittelalter, Frankfurt a.M. 2006; PITZ, Bürgereinung (wie Anm. 7), S. 418-441. 
Peter Blickle zum Modell seiner Kommunalismus-These gemacht hat ${ }^{10}$, keine Spezifika der Entwicklung im Reich im 14. und 15. Jahrhundert gewesen sind. Doch sind Formen »konsensualer Herrschaft « ${ }^{11}$ im spätmittelalterlichen Reich besonders vielfältig und mit Blick auf die Entwicklung in der Frühen Neuzeit auch zukunftsweisend geworden.

Gerade die Adelsgesellschaften zeichnete ein großes Spektrum unterschiedlicher Typen aus. Es reichte im 14. und 15. Jahrhundert von Erbeinungen, mit denen hochadelige Verwandtschaftsverbände langfristig organisiert werden konnten ${ }^{12}$, über Bruderschaften mit religiöser bzw. liturgischer Ausrichtung bis hin zu Turniergesellschaften, die im Reich adelige Standeskultur jenseits eines Fürstenhofes demonstrierten ${ }^{13}$, oder Hoforden. Politisch dienten adelige Zusammenschlüsse der Selbstbehauptung in regionalen bzw. interterritorialen Kontexten und gewannen in ihren bedeutendsten Ausformungen wie der Gesellschaft mit Sankt Georgenschild in Süddeutschland schließlich auch reichsverfassungsgeschichtliche Bedeutung. Es waren im Übrigen gerade Adelseinungen wie der Sankt Georgenschild, die die anspruchsvollsten Modelle interner Friedenswahrung entwickelten, was angesichts der Verbreitung adeliger Fehde wiederum nicht überrascht ${ }^{14}$.

Besonders die Adelsorganisationen im Reich demonstrierten, dass genossenschaftliche Organisationsformen mit der hierarchisch gegliederten Ständeordnung vereinbar waren: So sahen sich die hochadeligen, da »edelfreien « Grafen und Herren vor das Problem gestellt, beim Konnubium einerseits an der ständischen Abgrenzung zum Niederadel festzuhalten ${ }^{15}$, sich andererseits

10 Peter Blickle, Kommunalismus - Begriffsbildung in heuristischer Absicht, in: DERS. (Hg.), Landgemeinde und Stadtgemeinde in Mitteleuropa: Ein struktureller Vergleich, München 1991, S. 5-38; DerS., Kommunalismus, 2 Bde., München 2000.

11 Bernd SCHNEIDMÜLLER, Konsensuale Herrschaft. Ein Essay über Formen und Konzepte politischer Ordnung im Mittelalter, in: Paul-Joachim HeINIG, Sigrid JAHNS, Rainer Christoph Schwinges, Sabine WefERS (Hg.), Reich, Regionen und Europa in Mittelalter und Neuzeit. Festschrift für Peter Moraw, Berlin 2000, S. 53-87.

12 Die umfassendste Übersicht bietet immer noch Johann Jacob Moser, Teutsches Staatsrecht, 17. Theil, Leipzig 1745. S. 9-169.

13 Andreas RANFT, Die Turniere der vier Lande: Genossenschaftlicher Hof und Selbstbehauptung des niederen Adels, in: Zeitschrift für die Geschichte des Oberrheins 142, Neue Folge 103 (1994), S. 83-102.

${ }^{14}$ Herbert OBEnAUS, Recht und Verfassung der Gesellschaften mit St. Jörgenschild in Schwaben. Untersuchungen über Adel, Einung, Schiedsgericht und Fehde im fünfzehnten Jahrhundert, Göttingen 1961; zum Fehdewesen zusammenfassend Christine REINLE, Fehde, in: Handwörterbuch zur deutschen Rechtsgeschichte, 2. Aufl., hg. von Albrecht CORDES, Heiner LÜCK u.a., München 2007, Sp. 1514-1525.

15 Karl-Heinz SpIEsS, Ständische Abgrenzung zwischen Hochadel und Ritteradel im Spätmittelalter, in: Rheinische Vierteljahrsblätter 56 (1992), S. 181-205, hier S. 188190. 
aber zur Behauptung ihres ständischen Ranges auf gemeinsame Einungen mit ebendiesem Niederadel einzulassen und die damit einhergehenden egalitären Konsequenzen einer solchen gemeinsamen Vergesellschaftung ausbalancieren zu müssen ${ }^{16}$.

Zweifellos kompensierten solche Einungen - seien es Adels- oder Städtebünde - vor allem im 15. Jahrhundert strukturelle Schwächen einer noch wenig verdichteten Reichsverfassung oder nutzten regionale Freiräume, die sich aufgrund schwacher oder konkurrierender fürstlicher Herrschaften ergaben ${ }^{17}$. Eine strikte Opposition zur Herrschaftspraxis der Mächtigeren lag gleichwohl nicht vor, denn die Übergänge von adeligen Einungen zur landständischen Repräsentation waren häufig fließend. Vielfach basierten die landständischen Repräsentationsformen in den Territorien des Reiches auf früheren Einungen des Adels. Im Reich selbst wiederum trugen Einungen entscheidend zur »Verdichtung « politischer Kommunikation bei. Das prominenteste und wichtigste Beispiel dafür ist die exklusive Kurfürsteneinung, die mit der Goldenen Bulle von 1356 zur tragenden Säule der politischen Ordnung des Reiches avancierte ${ }^{18}$. Aber auch die anderen Einungen des Adels erlangten in der Folgezeit reichsrechtliche Legitimation: Nachdem die Goldene Bulle 1356 zunächst mit Ausnahme der Kurfürsten andere Adelseinungen noch prinzipiell verboten hatte, weil sie nicht dem Landfrieden dienten (was faktisch zweifellos seine Richtigkeit hatte), wurden im Gefolge der Appenzellerkriege 1406 erstmals Adelsbünde vom König legitimiert ${ }^{19} .1422$ wurde diese reichsrechtliche Legitimation dann mit einem Privileg König Sigismunds auf alle Adelseinungen ausgedehnt, soweit sie sich dem Schutz des Landfriedens verpflichteten. In der Realität blieb dies zwar häufig deklaratorisch, aber es entwickelte sich damit im Reich eine Tradition, Einungen - und gerade auch Adelseinungen - als reichsrechtlich legitimierte Form der organisierten Friedenswahrung und -sicherung anzuerkennen.

16 Horst CARL, Einungen und Bünde. Zur politischen Formierung des Reichsgrafenstandes im 15. und 16. Jahrhundert, in: Kurt Andermann, Clemens Joos (Hg.), Grafen und Herren in Südwestdeutschland vom 12. bis ins 17. Jahrhundert, Epfendorf 2006, S. 97-118, hier S. 102-105.

17 Diesen Surrogatcharakter betont v.a. Peter MoRAw, Die Funktion von Einungen und Bünden im spätmittelalterlichen Reich, in: Volker Press, Dieter STIEVERMANN (Hg.), Alternativen zur Reichsverfassung in der Frühen Neuzeit, München 1995, S. 1-21.

18 Bernd-Ulrich Hergemüller, Fürsten, Herren, Städte zu Nürnberg 1355/56. Die Entstehung der »Goldenen Bulle« Karls IV., Köln 1983.

19 Johann Stephan BurgermeISTER, Codex diplomaticus equestris cum continuatione oder Reichs-Ritterschaftliches Archiv [...], 2 Bde., Ulm 1721, Bd. 1, S. 1-12. 
Unabhängig von der ständischen Zusammensetzung funktionierte ein Landfriedensbund ${ }^{20}$ als ein System kollektiver Sicherheit in Form einer Schwureinung. Es war gleichsam eine $»$ Verschwörung zum Frieden $\aleph^{21}$, um das Fehdewesen überflüssig zu machen und Frieden zu schaffen. Erreicht werden sollte dies dadurch, dass sich alle Mitglieder per Eid auf Verfahren der Friedenssicherung nach innen wie nach außen verpflichteten. Für Konflikte der Mitglieder untereinander wurde eine Schiedsgerichtsbarkeit eingeführt, während der Bund nach außen die Verteidigung seiner Mitglieder in die Hand nahm. Um Geld für solche Kriegsleistungen zur Verfügung zu haben, erstreckte sich die Selbstverpflichtung der Einungsmitglieder auch auf die Finanzierung mittels Steuerbeiträgen. Schließlich organisierten die Bundesmitglieder regelmäßige Beratungs- und Entscheidungsgremien in Form eines Bundesrates mit Bundeshauptleuten an der Spitze.

Freilich konnte eine ständische Einung das Problem der Friedenswahrung gerade in den zersplitterten Kernregionen des Reiches nicht lösen, denn für eine flächendeckend orientierte Landfriedensregelung bedurfte es ständeübergreifender Zusammenschlüsse. Hier kommt nun der Schwäbische Bund ins Spiel, weil mit ihm ein ständeübergreifendes System kollektiver Sicherheit auf der Basis einer Landfriedenseinung geschaffen wurde, der im und für das Heilige Römische Reich Modellcharakter erhalten sollte22.

\section{Der Schwäbische Bund als ständeübergreifendes Modell genossenschaftlicher Friedenswahrung}

Mit der Gründung des »kaiserlichen Bundes im Lande Schwaben« knüpfte Kaiser Friedrich III. (1440-1493) an die spätmittelalterliche Landfriedenspolitik des Königtums an, verband diese aber geschickt mit den autochthonen

20 Allgemein Guido Komatsu, Landfriedensbünde im 16. Jahrhundert. Ein typologischer Vergleich, Dissertation, Universität Göttingen 2001, S. 8-16, 209-242.

${ }^{21}$ Otto Gerhard OeXle, Friede durch Verschwörung, in: Johannes Fried (Hg.), Träger und Instrumentarien des Friedens im Hohen und Späten Mittelalter, Sigmaringen 1996, S. 114-150.

22 Die Charakterisierung des Schwäbischen Bundes als »System kollektiver Sicherheit« geht auf Volker Press zurück: Volker PrESS, Die Bundespläne Karls V. und die Reichsverfassung, in: DeRS., Das Alte Reich. Gesammelte Aufsätze, hg. v. Johannes Kunisch, unter Mitarbeit von Horst CARL, Gabriele HAUG-MoritZ u.a., Berlin 1997, S. 67-127, hier S. 70, 111. 
regionalen Traditionen genossenschaftlicher Landfriedenswahrung ${ }^{23}$. In einer krisenhaften Zuspitzung der politischen Situation gelang es ihm 1488, die Mindermächtigen des Landes Schwaben - Reichsstädte und adelige Georgenschild-Gesellschaften - zum zunächst auf acht Jahre befristeten Zusammenschluss zur Wahrung des Frankfurter Reichslandfriedens von 1486 zu bewegen. Er konnte dabei davon profitieren, dass in Schwaben bereits eine lange Tradition genossenschaftlicher Zusammenschlüsse sowohl beim Adel als auch auf Seiten der zahlreichen Reichsstädte existierte. Neu war jedoch deren ständeübergreifender Zusammenschluss in einem gemeinsamen Landfriedensbund. Der Kaiser sah sich dazu umso mehr berechtigt, als die schwäbischen Städte und Adeligen nach seiner Ansicht keinem eigenen Fürsten untertan, sondern unmittelbar Kaiser und Reich verpflichtet seien. Was zunächst politisch als Sammlung einer prohabsburgischen Klientel intendiert war, erwies sich schließlich als Weichenstellung von beträchtlicher verfassungspolitischer Fernwirkung, handelte es sich doch bei Schwaben um eine zentrale »königsnahe « ${ }^{24}$ Landschaft im Reich. Der erfolgreiche Zusammenschluss entwickelte zudem rasch eine Eigendynamik, die ihn zu einem eigenständigen politischen Faktor im Reich werden ließ, denn noch in der Anfangsphase schlossen sich mit Tirol, Württemberg, den Erzbischöfen von Trier und Mainz und den fränkischen und badischen Markgrafen mächtige Reichsfürsten an. Sowohl in der ständischen Zusammensetzung als auch der regionalen Ausdehnung griff der Landfriedensbund damit weit über den ursprünglichen Rahmen aus. Da seit 1490 zudem König Maximilian als Landesherr Tirols und der Mainzer Kurfürst Berthold von Henneberg dem Bund angehörten, vereinte der Schwäbische Bund auch die Protagonisten und zeitweiligen Gegenspieler des Verfassungswandels im Reich, der als »Reichsreform« bezeichnet wird, unter seinem Dach.

Im Unterschied zur Eidgenossenschaft, der zweiten großen Landfriedenseinung in Mitteleuropa um $1500^{25}$, war das schwäbische Pendant jedoch nicht

23 Horst CARL, Der Schwäbische Bund 1488-1534. Landfrieden und Genossenschaft im Übergang vom Spätmittelalter zur Reformation, Leinfelden-Echterdingen 2000, S. 21-148.

${ }^{24}$ Als Kategorie eingeführt von Peter Moraw, Franken als königsnahe Landschaft im späten Mittelalter, in: Blätter für deutsche Landesgeschichte 112 (1976), S. 123-138.

25 Zum ursprünglichen Charakter der schweizerischen Eidgenossenschaft als Landfriedensbund grundlegend Peter BLICKLE, Friede und Verfassung. Voraussetzungen und Folgen der Eidgenossenschaft von 1291, in: Innerschweiz und frühe Eidgenossenschaft. Jubiläumsschrift 700 Jahre Eidgenossenschaft, Olten 1991, Bd. 1, S. 15202. Die verfassungsgeschichtlichen Parallelen zwischen Eidgenossenschaft und Schwäbischem Bund werden gerade in der Schweizer Historiographie meist aufgrund der unterschiedlichen ständischen Zusammensetzung unterschätzt. Verwiesen sei allein schon auf die starke Stellung der reichsunmittelbaren Städte in beiden Bünden, 
auf ewig geschlossen, sondern immer nur auf eine begrenzte Zeit. Er musste dann jeweils nach langwierigen und nervenaufreibenden Verhandlungen verlängert werden. Entsprechende »Erstreckungen« des Bundes fanden 1496, 1500, 1512 und 1523 statt. Diese Verlängerungen boten nicht nur neuen Mitgliedern die Möglichkeit, dem Bund beizutreten, sie erlaubten es auch Bundesmitgliedern, sich einer Fortsetzung zu entziehen. Auch diese Mitgliederfluktuation unterscheidet ihn von den Eidgenossen.

In seiner letzten Phase (1523-1534) gehörten dem Bund alle wichtigen Territorien Oberdeutschlands von der Kurpfalz bis zum Erzbistum Salzburg an (s. Karte »Bundesstände 1523-1534«, S. 138). Er behielt aber seinen schwäbischen Charakter, weil die schwäbischen Reichsstädte und Teile des schwäbischen Adels im Bund bis zu seinem Ende präsent blieben. Da der Bund ab 1500 in drei ständisch gegliederte »Bänke« (Fürsten, Adel, Städte) organisiert war, wobei die Vertreter von Städten und Adel jeweils eine Bank besetzten, verblieb den schwäbischen Mindermächtigen trotz der Mitgliedschaft mächtiger Fürsten eine starke Position im Bund. Ein vergleichbares Gegengewicht gegen die Fürsten haben Städte und reichsunmittelbarer Adel beispielsweise auf dem Reichstag nie ausbilden können.

Sorgte der Landfriedensbund mit seinem obligatorischen Schiedsgericht dafür, dass die Mitglieder ihre Konflikte vor dem Bundesgericht, das von 1501 bis 1513 den berühmten Humanisten Reuchlin zu seinen Richtern zählte ${ }^{26}$, rechtlich beilegten, und ist er damit seiner Aufgabe der internen Friedenswahrung seiner Mitglieder in hohem Maße gerecht geworden, so erzielte er seine spektakulärsten Erfolge doch in den militärischen Auseinandersetzungen mit seinen äußeren Widersachern, angefangen von den bayerischen Wittelsbachern 1492, Herzog Ulrich von Württemberg 1519, dem fränkischen Fehdeadel 1523 bis hin zu den aufständischen Bauern im Bauernkrieg $1525^{27}$.

vgl. Horst CARL, Eidgenossen und Schwäbischer Bund - feindliche Nachbarn?, in: Peter RÜCK (Hg.), Die Eidgenossen und ihre Nachbarn im Deutschen Reich des Mittelalters, Marburg 1991, S. 215-265.

${ }^{26}$ Horst CARL, »Triumvir Sueviae« - Reuchlin als Bundesrichter, in: Reuchlin und die politischen Kräfte seiner Zeit, hg. von Stefan RHEIN, Sigmaringen 1998, S. 65-86; Ders., Schwäbischer Bund (wie Anm. 23), S. 398-401; Markus Rafael ACKermanN, Der Jurist Johannes Reuchlin (1455-1522), Berlin 1999, S. 107-148.

27 CARL, Schwäbischer Bund (wie Anm. 23), S. 431-496. 


\section{Bund und Reich}

Nicht nur personell, sondern auch institutionell beeinflusste der schwäbische Landfriedensbund die Diskussion um die Reformansätze des Wormser Reichstages 1495, und wichtige Reformimpulse wie die Verkündung des Ewigen Landfriedens, die Aufrichtung des Kammergerichts, die Periodizität des Reichstages oder die Diskussion um ein Reichsregiment profitierten von konkreten Erfahrungen des Landfriedensbundes. Die verfassungsgeschichtliche Bedeutung des Bundes liegt deshalb vor allem darin, dass in den für die Ausgestaltung der Reichsverfassung wohl entscheidenden Jahren zwischen 1490 und 1500 Kernregionen des Reiches in Form eines traditionellen Landfriedensbundes erfolgreich organisiert wurden.

Die Rolle des Schwäbischen Bundes für die Reformen des Wormser Reichstages ist von der älteren Forschung in der Regel unterschätzt worden. Der Wormser Reichstag war gekennzeichnet durch ein Tauziehen zwischen Kaiser Maximilian und den Reichsständen, der Schwäbische Bund aber wenige Jahre zuvor 1488 durch Maximilians Vater, Kaiser Friedrich III., ja als kaiserlicher Bund per Mandat ins Leben gerufen worden. Wenn man ihn deshalb der kaiserlichen Seite zuordnete - und in der Tat hatte er in den Jahren zwischen 1488 und 1495 entscheidend zu politischen Erfolgen der Habsburger im Reich beigetragen -, dann konnte man ihn nur schwer in den sich immer deutlicher herausbildenden Dualismus von Kaiser und Reich verorten $^{28}$. Die Parallelität mit den Problemen im Reich war jedoch evident: Auch beim Wormser Reichstag 1495 ging es darum, die überbordende Friedlosigkeit in den Griff zu bekommen und dazu die Reichsstände von den Fürsten bis hin zu den Reichsstädten in die Pflicht zu nehmen. Und auch in Worms musste dies einhergehen mit institutionellen Lösungen für eine zentrale Gerichtsbarkeit, die eine gewaltsame Selbsthilfe mittels Fehde obsolet machte, und für eine Finanzierung des Reiches, die die Mittel für Verteidigung und Kriegführung bereitstellte.

Im Zentrum dieses Verfassungswandels stand folglich der sogenannte »Ewige Landfrieden«, und an diese Errungenschaft kristallisierten sich die anderen Verfassungslösungen Reichskammergericht und Reichstag an ${ }^{29}$.

28 Weil er den Bund auf Seiten des Königs verortet, ist die Bedeutung des Bundes für das Agieren der Reichsstände vor allem dem Autor der klassischen Darstellung der sogenannten Reichsreform entgangen: Heinz ANGERMEIER, Die Reichsreform 1410 1555. Die Staatsproblematik in Deutschland zwischen Mittelalter und Gegenwart, München 1984, S. 169.

${ }_{29}$ Mattias G. FiSCHER, Reichsreform und Ewiger Landfrieden, Aalen 2007 (Untersuchungen zur deutschen Staats- und Rechtsgeschichte, 34); erst die sog. »Handhabung« - die Exekution des Landfriedens -, die 1495 dem Reichstag überantwortet wurde, 
Dieser Ewige - will heißen unbefristete - Landfrieden bedurfte der »Exekution«, oder wie es im Reichsabschied hieß, der »Handhabung Friedens und Rechts«. Da dafür der Reichstag zuständig sein sollte, war dies eine entscheidende Wegmarke für die Verselbständigung und Periodizität dieses zentralen Reichsorgans, das sich damit aus der Aura des königlichen Hoftags emanzipieren konnte. Und bezeichnenderweise war diese Exekution des Landfriedens auch in den Beschlüssen des Wormser Reichstages 1495 dem Wortlaut nach als Einung, als Vereinbarung zwischen König und Ständen, und nicht als königliche Setzung formuliert. Es war ebenfalls kein Zufall, dass nach 1512 die Reichskreise, in denen die regionalen genossenschaftlichen Strukturen des spätmittelalterlichen Reiches fortlebten, mit der Wahrung des Landfriedens betraut wurden. Mit der Reichsexekutionsordnung von 1555 wurde dies auf Dauer institutionalisiert und blieb bis zum Ende des Alten Reiches Grundlage der Landfriedenswahrung.

Die Parallelen zwischen Schwäbischem Bund und den Errungenschaften des Wormser Reichstages von 1495 sind jedoch nicht nur institutionell und funktional gewesen. Wir können die Leitfiguren der ständischen Opposition gegen Maximilian identifizieren, weil Maximilian mehrfach im Nachhinein seinem Zorn über sie Luft gemacht hat und einige am liebsten aufs Schafott gebracht hätte. Die Personen, die er nannte - Kurfürst Berthold von Henneberg, Herzog Eberhard von Württemberg und Graf Haug von Werdenberg gehörten zugleich aber auch dem Führungszirkel des Schwäbischen Bundes an, Haug von Werdenberg etwa war 1488 der eigentliche spiritus rector der Bundesgründung gewesen ${ }^{30}$.

Mein Argument ist also, dass der 1495 ausgestaltete Verfassungskompromiss zwischen Kaiser und Ständen Traditionen des spätmittelalterlichen Einungswesens aufgenommen bzw. für das Reich adaptiert hat. Das Reich ist kein Bund, keine Assoziation gewesen und auch in der Frühen Neuzeit nicht geworden, aber es hat, um überleben zu können, doch in wesentlichen Segmenten wie eine Einung der Stände funktioniert bzw. Erfahrungen des Einungswesens in seiner avanciertesten Form - eben dem Schwäbischen Bund - übernommen bzw. fortgeführt ${ }^{31}$.

erforderte dessen periodisches Zusammentreten und förderte folglich die institutionalisierte Periodizität.

${ }^{30}$ In Worms war Haug von Werdenberg als württembergischer Landhofmeister präsent, somit nicht in königlichen, sondern fürstlichen Diensten. Angermeier hat Werdenberg, der in den 1480er Jahren der engste Berater Friedrichs III. gewesen war, falsch zugeordnet. ANGERMEIER, Reichsreform (wie Anm. 28), S. 169; zum Personaltableau der Wormser Reformpartei vgl. CARL, Schwäbischer Bund (wie Anm. 23), S. 78-80.

31 Horst CARL, Einungen und Bünde, in: Stephan Wendehorst, Siegrid WestPHAL (Hg.), Lesebuch Altes Reich, München 2006, S. 101-106. 


\section{Frieden durch Verschwörung Die Realität bündischer Landfriedenswahrung}

Wenn man nun den Schwäbischen Bund daran misst, wie er denn seine eigentliche Aufgabe, die Wahrung bzw. Durchsetzung des Landfriedens, gelöst hat, so hängt seine recht positive Einschätzung nicht zuletzt mit ebendiesen Erfolgen in der Landfriedenswahrung zusammen. Allerdings hat er sich strikt auf diese Funktionen beschränkt, so dass er ähnlich wie die Eidgenossenschaft keine Entwicklung in Richtung auf ein frühneuzeitliches Staatswesen eingeschlagen hat.

Erfolge lassen sich vor allem in dem Bereich festmachen, für den er ursprünglich gegründet worden ist, nämlich für die allenthalben grassierenden Adelsfehden. Der fehdeführende Adel galt noch zur Zeit der Bundesgründung 1488 als Hauptverursacher der Friedlosigkeit im Reich, und dass dieses Problem im Reich beim Auslaufen des Bundes 1534 weitgehend gelöst erschien, hing auch mit dem Wirken des Bundes in seinem Einzugsgebiet zusammen. Götz von Berlichingen - der bis heute populärste Vertreter dieser Gattung aufgrund von Goethes Drama - fiel 1519 dem Bund in die Hände und wurde durch lebenslange Urfehde neutralisiert ${ }^{32}$, doch spektakulärer noch war der Strafzug des Bundes 1523 gegen die besonders berüchtigten und aktiven fränkischen Fehderitter ${ }^{33}$. Nach der Zerstörung bzw. Entfestigung von 24 Adelsburgen war den Protagonisten der Adelsfehde in Franken das Rückgrat gebrochen. Gerade die spätmittelalterliche fränkische Ritterschaft, die in den letzten Jahren besonders intensiv erforscht worden ist ${ }^{34}$, scheint genau das Urteil, das Philippe Contamine 1997 beim Vergleich des deutschen mit

32 Helgard UlmschneIder, Götz von Berlichingen. Ein adeliges Leben der deutschen Renaissance, Sigmaringen 1974, S. 108-111.

33 Peter RitZmanN, »Plackerey in teutschen Landen«. Untersuchungen zur Fehdetätigkeit des fränkischen Adels im frühen 16. Jahrhundert und ihrer Bekämpfung durch den Schwäbischen Bund und die Reichsstadt Nürnberg, insbesondere am Beispiel des Hans Thomas von Absberg und seiner Auseinandersetzungen mit den Grafen von Oettingen (1520-1531), Diss. München 1995, S. 240-380; Christine REINLE, Fehden und Fehdebekämpfung am Ende des Mittelalters. Überlegungen zum Auseinandertreten von »Frieden« und »Recht« in der politischen Praxis zu Beginn des 16. Jahrhunderts am Beispiel der Absberg-Fehde, in: Zeitschrift für Historische Forschung 30 (2003), S. 355-388.

34 Joseph MORSEL, »Das sy sich mitt der besstenn gewarsamig schicken, das sy durch die widerwertigen Franckenn nitt nidergeworffen werdenn«. Überlegungen zum sozialen Sinn der Fehdepraxis am Beispiel des mittelalterlichen Franken, in: Dieter RÖDEL, Joachim SchNEIDER (Hg.), Strukturen der Gesellschaft im Mittelalter. Interdisziplinäre Mediävistik in Würzburg, Wiesbaden 1996, S. 140-167; Hillay ZMORA, State and Nobility in Early Modern Germany. The Knightly Feud in Franconia, 14401567, Cambridge 1997. 
dem französischen Adel gefällt hat, zu bestätigen - »que la noblesse française est beaucoup moins violente et beaucoup moins opposée aux villes et aux sociétés urbaines que la noblesse allemande $\aleph^{35}$. Im Vergleich zum französischen Adel sei der deutsche also städtefeindlicher, anarchischer und friedloser, alles in allem also: rückständiger gewesen. Doch dieses für das 15. Jahrhundert sicherlich verallgemeinerbare Urteil erscheint für das 16. Jahrhundert und speziell für das Einzugsgebiet des Schwäbischen Bundes nicht mehr zutreffend. Schließlich standen in den Auseinandersetzungen mit Götz von Berlichingen und den fränkischen Standesgenossen die schwäbischen Adeligen auf Seiten der Landfriedenseinung und identifizierten sich im Verbund mit den Reichsstädten somit mit der Ordnungsmacht, die nicht anders als die Reichsgesetzgebung des Ewigen Landfriedens die Selbsthilfe der Fehde obsolet machte ${ }^{36}$.

\section{Der Bauernkrieg von 1525}

Wenn der Schwäbische Bund jedoch überhaupt für eine politische Tat im kollektiven Gedächtnis der Deutschen haften geblieben ist, dann ist dies die brutale Liquidation der aufständischen Bauern durch sein Heer 1525 unter Führung des Georg Truchsess von Waldburg. Der Schwäbische Bund war unbestreitbar der wichtigste militärische Kontrahent der Bauern und damit für die militärische Niederschlagung des Bauernkrieges verantwortlich. Aber auch in diesem Kontext plädiere ich für eine etwas differenziertere Einschätzung des Agierens des Bundes, als wir es beispielsweise bei Peter Blickle oder anderen klassischen Darstellungen zum Bauernkrieg finden ${ }^{37}$. Dazu muss allerdings etwas weiter ausgeholt werden: In das Schema eines traditionellen Landfriedensbundes, der Landfriedensbruch entweder bei kriminellen

35 Philippe Contamine, La noblesse au royaume de France de Philippe le Bel à Louis XII. Essai de synthèse, Paris 1997, S. 331.

${ }^{36}$ Horst CARL, Fränkische Unruhestifter und schwäbische Ordnungshüter? Schwäbisches und fränkisches Regionalbewusstsein im Kontext frühneuzeitlicher Politik, in: Thomas KÜHnE, Cornelia RAUH-KüHnE (Hg.), Raum und Geschichte. Regionale Traditionen und föderative Ordnungen von der Frühen Neuzeit bis zur Gegenwart, Festschrift Bernhard Mann, Leinfelden-Echterdingen 2001, S. 24-37; Ders., Genossenschaft und Herrschaftsverdichtung - zur politischen Kultur von Adelseinungen im Alten Reich, in: Ronald Asch, Dagmar FreIST (Hg.), Staatsbildung als kultureller Prozess. Strukturwandel und Legitimation von Herrschaft in der Frühen Neuzeit, Köln, Weimar, Wien 2005, S. 405-427, hier S. 413-415.

37 Peter Blickle, Die Revolution von 1525, München ${ }^{4}$ 2004; Ders., Der Bauernkrieg. Die Revolution des Gemeinen Mannes, München ${ }^{3} 2006$. 
sogenannten »landschädlichen Leuten« oder eben bei adeligen Fehdegegnern verfolgt, ließen sich aufrührerische Untertanen nur schwer einordnen; sie fehlten deshalb auch noch im Kontext des Ewigen Landfriedens von 1495 als Problemgruppe, obwohl sich seit den 1470er Jahren die Untertanenunruhen im Reich signifikant häuften. Die Herrschenden konnten jedoch ihre Augen nicht vor den immer mehr um sich greifenden Unruhen als Herausforderung für die Friedenssicherung verschließen, und deshalb kam es 1500 erstmals zur Aufnahme eines gänzlich neuen Artikels in die Bundesordnung. Damit die Untertanen nicht gewaltsamen Aufruhr übten, wurde nunmehr den Gemeinden die Möglichkeit eingeräumt, Klagen gegen ihre Herrschaften vor die Bundesversammlung zu bringen, die dann mittels eines Schiedsverfahrens zwischen Herrschaft und Untertanen vermitteln sollte. Der Bund regelte in diesem Verfahren nicht die Einzelheiten, sprach also nicht materielles Recht, sondern regelte die Zusammensetzung eines Schiedsgerichtes, vor dem die Streitpunkte verhandelt wurden - die Maxime entsprach der des Ewigen Landfriedens, dass der Rechtsweg eröffnet werden muss, um offene Gewalt und Aufruhr zu verhindern.

Die Tätigkeit des Bundes als Schiedsinstanz in Untertanenunruhen ist durchaus nicht hypothetisch geblieben, die beiden bekanntesten Fälle Kempten (1492) und Ochsenhausen (1502) sind bevorzugtes Objekt der Forschungen von Peter Blickle geworden ${ }^{38}$. Am Ende der Auseinandersetzung stand jeweils ein vom Bund garantierter Schiedsvertrag, der den Untertanen in einem entscheidenden Punkt entgegenkam: Er fixierte die Herrenrechte und machte deren Übertretung rechts- bzw. sanktionsfähig, so dass die Untertanen bei Übertretung des garantierten Vertrages an den Bund appellieren und mit Rechtshilfe rechnen konnten ${ }^{39}$.

Es erscheint angesichts der Schlächtereien des Bauernkrieges paradox, wenn diese positive Kontinuitätslinie einer Verbesserung der Stellung der Untertanen durch die bündische Schiedsgerichtsbarkeit bis ins Jahr 1525 gezogen wird. Aber der Weingartner Vertrag, den der Truchsess Georg III. von Waldburg am 17. April 1525 mit den oberschwäbischen Bauern abschloss, gehört in diese Tradition bündischer Schiedstätigkeit. Der Weingartner Vertrag war weder eine bedingungslose Kapitulation der Bauern, noch haben die Herren hier die Bauern übervorteilt. Nimmt man die Zusammensetzung des im Vertrag für die Einzelklagen vorgesehenen Schiedsgerichtes, so haben die Bauern nie ein günstigeres Schiedsgericht zugesprochen bekommen, denn dieses bestand nur aus Reichsstädtern, von denen die Bau-

38 Peter BlickLe, Landschaften im Alten Reich. Die staatliche Funktion des Gemeinen Mannes in Oberdeutschland, München 1973, S. 112-116, 334-338.

39 CARL, Schwäbischer Bund (wie Anm. 23), S. 485-489; Thomas F. SEA, The Swabian League and Peasant Disobedience before the German Peasants' War of 1525, in: Sixteenth Century Journal 30 (1999), S. 89-111. 
ern mit gutem Grund eine Entscheidung zu ihren Gunsten erhoffen konnten ${ }^{40}$. Diese Regelung hat allerdings nur im oberschwäbischen Kernland des Bundes, in dem es keine dominierenden fürstlichen Territorien gab, funktioniert. Schon in Württemberg und mehr noch in Franken ist der Bund im Sinne der Fürsten nur noch als militärische Ordnungsmacht tätig geworden. Wenn die oberschwäbischen Untertanen sich mit dem Weingartner Vertrag jedoch auf die grundlegenden Spielregeln der Landfriedenswahrung durch den Bund einließen, dann hatte dies nachhaltige Konsequenzen: Der Rechtsweg schloss bewaffneten Widerstand aus, und das »göttliche Recht« bzw. die reformatorischen Forderungen waren im bündischen Schiedsverfahren nicht verhandelbar, wohl aber die Wahrung eines rechtlich gesicherten Status quo. Auf dieser Grundlage kam eine zutiefst konservative Einigung zustande, die die Bauern gegen eine Verschlechterung ihrer Rechtslage sicherte, zugleich aber auch dafür sorgte, dass Oberschwaben eine altgläubige Region blieb ${ }^{41}$.

Es ist dieses Erbe - Verrechtlichung sozialer Konflikte ${ }^{42}$ und gleichzeitige Wahrung des Status quo -, das der Landfriedensbund somit über den Bauernkrieg hinweg an das Reich weitertradiert hat ${ }^{43}$. Hier aber hat es ab den 1570er Jahren bei den höchsten Reichsgerichten eine ganz erhebliche Rolle gespielt, dass Bauerngemeinden gegen ihre Herren vor den höchsten Reichsgerichten mit Aussicht auf Erfolg klagen konnten - ein Umstand, der in der neueren Frühneuzeit-Forschung eine bedeutende Rolle für die Aufwertung des Alten Reiches gespielt hat ${ }^{44}$.

40 Horst CARL, Landfriedenseinung und Ungehorsam - der Schwäbische Bund in der Geschichte des vorreformatorischen Widerstandsrechts im Reich, in: Robert vON FRIEDEBURG (Hg.), Widerstandsrecht in der frühen Neuzeit. Erträge und Perspektiven der Forschung im deutsch-britischen Vergleich, Berlin 2001 (ZHF Beiheft 26), S. 85112, hier S. 109-111.

41 Vadim Oswalt, Staat und ländliche Lebenswelt in Oberschwaben 1810-1871. (K)ein Kapitel im Zivilisationsprozess?, Leinfelden-Echterdingen 2000.

42 Als wesentliches Merkmal des Reiches im Vergleich mit anderen frühneuzeitlichen europäischen Gemeinwesen vor allem herausgestellt von Winfried Schulze, Die veränderte Bedeutung sozialer Konflikte im 16. und 17. Jahrhundert, in: DeRs. (Hg.), Europäische Bauernrevolten der Frühen Neuzeit, Frankfurt a.M. 1982, S. 276-308, v.a. S. $279,294 f$.

43 CARL, Landfriedenseinung und Ungehorsam (wie Anm. 40), S. $111 \mathrm{f}$.

44 Georg SchmidT, Geschichte des Alten Reiches. Staat und Nation in der Frühen Neuzeit 1495-1806, München 1999, S. 137-142. 


\section{Konfessionskonflikt und Landfriedenspolitik}

Dass mit dem Instrumentarium der Landfriedenspolitik schließlich auch die Konfessionsspaltung als größte Herausforderung des 16. Jahrhunderts wenn schon nicht bewältigt, so doch zumindest pazifiziert werden konnte, hat gleichfalls eine Wurzel in Verfahren des Landfriedensbundes ${ }^{45}$. Das bündische Schiedsverfahren war in erster Linie als vorläufiges Verfahren konzipiert, um die Selbsthilfe der Bundesgenossen einzuschränken. Es sollte verhindert werden, dass bei internen Streitigkeiten unter den Bundesmitgliedern die übliche Spirale von Gewalt und Gegengewalt in Gang gesetzt wurde. So sah die Bundesordnung seit 1496 vor, dass ein von anderen Mitgliedern angegriffenes oder geschädigtes Bundesmitglied kategorisch auf Selbsthilfe verzichten musste. Dies war vor allem für die Mächtigen, die Fürsten, eine Zumutung: Ein solcher Verzicht sei unerhört, bislang in Einungen nicht üblich und stelle einen Angriff auf seine fürstliche Standesehre dar, erklärte etwa 1513 Herzog Ulrich von Württemberg ${ }^{46}$, doch hielt der Schwäbische Bund an dieser Linie auch gegenüber den Fürsten fest. Der Bund schritt folglich in solchen Streitigkeiten zunächst mit dem Hinweis auf die beschworene Selbstverpflichtung der Mitglieder ein, um die Kontrahenten unter Androhung einer Bundesexekution auf den Rechtsweg zu zwingen. Damit aber war materiell über Recht oder Unrecht des Streits noch nichts entschieden, denn dies geschah erst im Hauptverfahren. Der Streitgegenstand selbst blieb also zunächst außen vor, und gerade dieser Umstand machte dieses Verfahren auch für Religionsstreitigkeiten anwendbar. Ab 1524 - also noch bevor dies auf Reichsebene beim Reichskammergericht geschah - klagten geistliche Fürsten vor Bundesversammlung und Bundesgericht gegen protestantische Mitstände aufgrund reformatorischer Eingriffe in ihre geistlichen Rechte. Die altgläubige Mehrheit der Bundesstände argumentierte dabei unter Berufung auf das positive Recht der Landfriedenseinung: Der Bund beanspruche ja gar keine Entscheidung in Wahrheitsfragen, weil in einem solchen Landfriedensverfahren doch nur die Kontrahenten wieder auf den Rechtsweg gezwungen würden und nicht vorab gewaltsam Fakten schaffen dürften. In den juristischen Verfahrenskategorien des Landfriedensrechts war auch die religiöse Wahrheitsfrage demnach eine Angelegenheit, die zunächst ausgeklammert werden konnte, weil sie erst in einem späteren Hauptverfahren zur Debatte stand.

Dieser Gedanke einer strikten Trennung von Friedenswahrung und religiöser Wahrheitsfrage als Versuch der Verrechtlichung der religiösen Auseinan-

45 Zum Folgenden CARL, Schwäbischer Bund (wie Anm. 23), S. 414-422; DerS., Landfriedenseinung und Ungehorsam (wie Anm. 40), S. 96-99.

${ }^{46}$ CARL, Triumvir (wie Anm. 26), S. 81. 
dersetzungen ließ sich im Bund nicht mehr realisieren, denn er wurde 1534 nicht mehr verlängert. In der anderen großen Landfriedenseinung der Zeit, der Schweizer Eidgenossenschaft, aber wurde genau dies 1531 mit dem Zweiten Kappeler Landfrieden verwirklicht. Der zu Kappel erneuerte Landfrieden besagte, dass jenseits des Status quo Religionsänderungen nur mehr auf gewaltfreiem Weg, also im Konsens der Bundesmitglieder erfolgen könnten; dieser Status quo aber wurde, wie es einer Landfriedenseinung entsprach, territorial definiert und auf die eidgenössischen Orte als Herrschaftsträger bezogen ${ }^{47}$. In der Tat wurden damit die kriegerischen Religionskonflikte in der Eidgenossenschaft für Generationen beendet, freilich um den hohen Preis, dass der territoriale Status quo geradezu unantastbar wurde und der Eidgenossenschaft die weitere Entwicklung zu einem geschlossenen Staatswesen erst einmal verbaut wurde.

Im Reich folgte man 1555 mit dem Augsburger Religionsfrieden ${ }^{48}$ genau diesem Modell: Die Reichsstände als Herrschaftsträger zogen die Konsequenz aus der Erfahrung, dass Religionsveränderung zum Krieg geführt hatte, das Ideal einer einheitlichen Konfession aber unrealistisch geworden war. Der Religionsfrieden propagierte Gewaltverzicht in Religionsfragen zwischen den Herrschaftsträgern und fixierte ihn dazu territorial. Der Religionsfrieden wurde im Übrigen ausdrücklich als Landfriedensregelung konzipiert, was der berühmte Augsburger Reichsabschied 1555 schon dadurch deutlich machte, dass sein Vertragstext zu drei Vierteln aus der neuen Reichsexekutionsordnung bestand, die nunmehr endgültig die Wahrung des Landfriedens im Reich in die Regie der Reichsstände bzw. der Reichskreise übertrug.

Der Schwäbische Bund hat keinen Bestand gehabt, er ist 1534 nicht verlängert worden, weil die ständischen Interessengegensätze seiner Mitglieder, namentlich aber die Konfessionskonflikte nicht mehr überbrückt werden konnten. Die Tatsache, dass er stets nur befristet war, führte dazu, dass in dieser kritischen Situation das Einigungspotential nicht mehr groß genug war, eine Verlängerung politisch durchzusetzen. In Gestalt des Heiligen Römischen Reiches stand jedoch eine politische Struktur zur Verfügung, die ebenso wie die Eidgenossenschaft unbefristet und für die eine Beendigung oder ein Austritt von Mitgliedern nicht vorgesehen war. Die Bundesmitglieder blieben somit weiterhin dem Reich verpflichtet, und die politischen Erfah-

47 Andreas WÜRGLER, »The League of Discordant Members« or How the Old Swiss Confederation Operated and How it Managed to Survive for so Long, in: André Holenstein, Thomas Maissen, Maarten PraK (Hg.), The Republican Alternative. The Netherlands and Switzerland Compared, Amsterdam 2008, S. 29-50, v.a. S. 39. 48 Axel GotThard, Der Augsburger Religionsfrieden, Münster 2004; Karl-Hermann KÄSTNER, Augsburger Religionsfriede, in: Handwörterbuch zur deutschen Rechtsgeschichte, 2. Auflage, hg. von Albrecht Cordes, Heiner LÜCK, Dieter WerkmüLLER u.a., Berlin 2008, Bd. 1, Sp. 360-362. 
rungen des Bundes blieben auch nach 1534 in diesem Reich mit seiner föderalen Struktur in einem geradezu Hegelschen Sinn »aufgehoben«.

Interpretiert man - wie ich dies getan habe - die Verfassungsgeschichte des Heiligen Römischen Reiches aus der Warte der im Schwäbischen Bund konkretisierten Tradition der kollektiven Landfriedenswahrung, dann stellen sich auch im Vergleich mit der französischen Monarchie die Defizite des Reichs auf dem Weg zu moderner Staatlichkeit im 16. Jahrhundert nicht mehr so eklatant dar, wie dies gemeinhin bei einem solchen Vergleich geschieht. Vergleicht man etwa die Rolle des Adels, so kann man das Urteil von Contamine über die Rückständigkeit des Adels im Reich durchaus relativieren: Durch die Adelsgesellschaften mit ihren Landfriedensbezügen ist der reichsunmittelbare Adel in Gestalt der Grafeneinungen und der Reichsritterschaft im Reich wahrscheinlich erfolgreicher pazifiziert worden als im Frankreich des 16. Jahrhunderts. Das Prinzip dieser Gesellschaften, durch Vereinbarung und Selbstverpflichtung der Mitglieder gewaltsamen Konfliktaustrag zu begrenzen bzw. auf den Rechtsweg zu bringen, dürfte im 16. Jahrhundert einer der entscheidenden Gründe für das Ende der Adelsfehden gewesen sein. Schließlich wurden so die potentiellen Urheber des Unfriedens selbst in die Pflicht genommen und konnten ihre adelige Revoltenneigung nicht so ungehemmt ausleben wie in Frankreich, wo dies geradezu als Spezifikum des Adels internalisiert wurde ${ }^{49}$.

In Frankreich scheiterte im Unterschied zur Eidgenossenschaft und zum Reich aber auch die Pazifizierung des Religionskonfliktes, was ab 1562 in die Katastrophe der Religionskriege mündete. Dieses Scheitern lag nicht zuletzt daran, dass es keine Tradition kollektiver ständeübergreifender Friedensregelung gab, um die Lücke, die das Königtum nach 1559 ließ, zu schließen. Ansätze hat es dazu durchaus gegeben - etwa auf munizipaler Ebene -, zum Tragen aber kamen sie nicht. Die französische ligue de Guise und ihre Verbündeten nach 1584 waren eben ein konfessionspolitisches Kampfbündnis und kein Landfriedensbündnis wie die ligue de Souabe ${ }^{50}$.

Wenn also die föderale Tradition im Reich durchaus wortwörtlich auf die Bünde (confédérations) zurückgeführt werden kann und auch das Reich in dieser Tradition vor allem ein System kollektiver Sicherheit dargestellt hat, ist damit zugleich - wie in der Eidgenossenschaft - auch der Preis, der für eine solche frühneuzeitliche »föderale« Ordnung zu zahlen war, markiert. Die Fixierung des Status quo war nicht nur Voraussetzung, sondern auch Konsequenz eines solchen Systems, das allenfalls in engen Grenzen reformierbar oder modernisierbar war.

49 Arlette Jounnna, Le devoir de révolte. La noblesse française et la gestation de l'État moderne, 1559-1661, Paris 1989; Ronald G. AsCH, Courtiers and Rebels: The Transformation of the European Nobilities, 1550-1700, London 2003, S. 106-122.

50 Jean-Marie Constant, La Ligue, Paris 1996. 


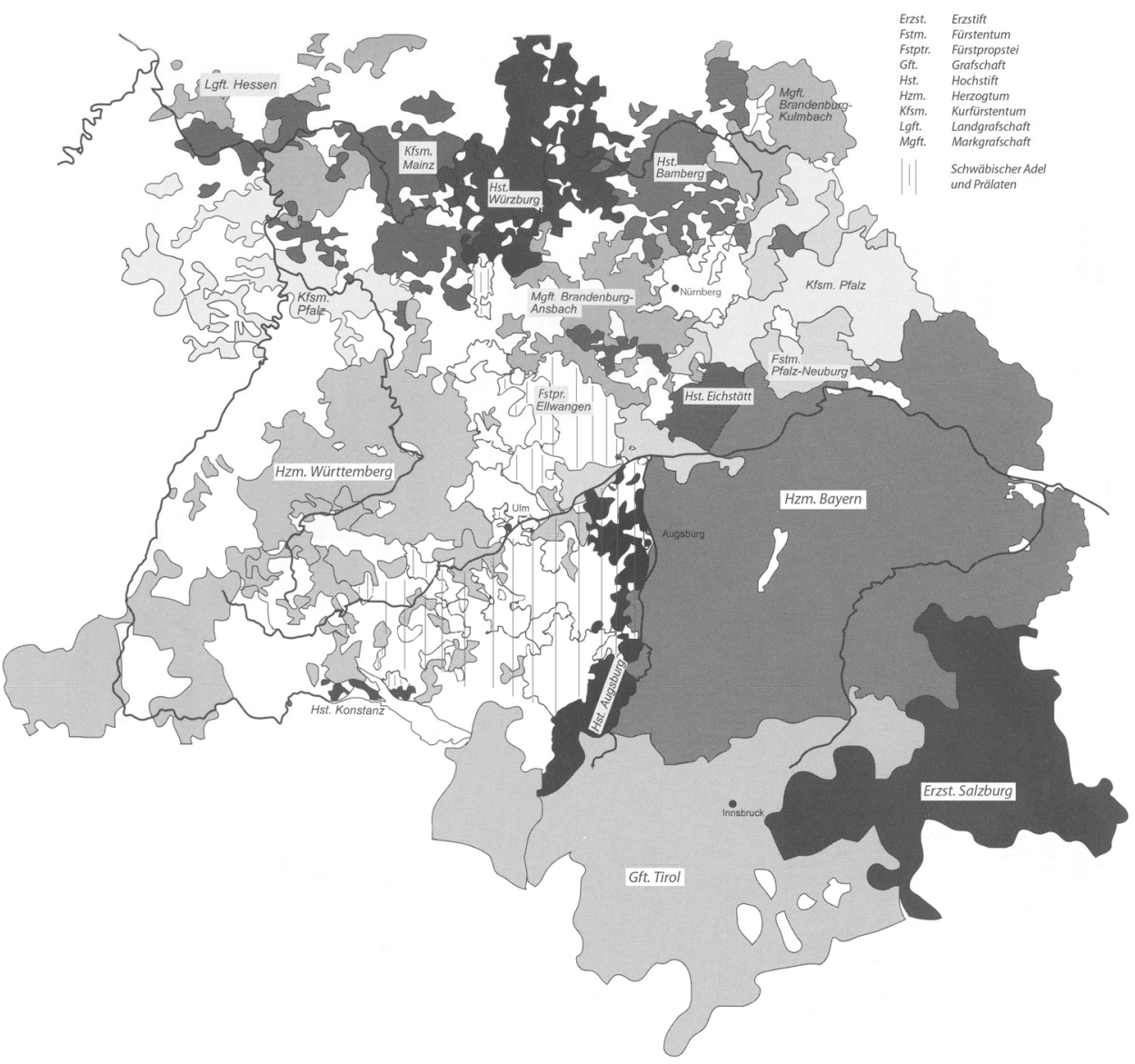

Bundesstände 1523-1534. Karte aus Horst CARL, Der Schwäbische Bund 1488-1534, Leinfelden-Echterdingen 2000, S. 66. Bearbeitung: Florian Roth 